\title{
The Heaviest Possible Ternary Trihalogen Species, IAtBr- Evidenced in Aqueous Solution: An Experimental Performance Driven by Computations
}

\author{
Ning Guo ${ }^{[a]}$, Dumitru-Claudiu Sergentu ${ }^{[a, b]}$, David Teze ${ }^{[a]}$, Julie Champion ${ }^{[a]}$, Gilles Montavon ${ }^{[a]}$, Nicolas \\ Galland $^{*,[b]}$ and Rémi Maurice ${ }^{*,[a]}$
}

\begin{abstract}
Evidencing new chemical species in solution is particularly challenging when one works at ultra-trace concentrations, as it is likely to happen with radioelements such as astatine $(Z=85)$. In this work, quantum mechanical calculations were used to predict the tiny experimental domain in which it is possible to detect the presence of an exotic ternary trihalogen anion, $\mathrm{IAtBr}^{-}$, and thus to guide a series of experiments. By analyzing the outcomes of competition experiments, we show that $\mathrm{IAtBr}^{-}$exists and can even predominate in aqueous solution. The equilibrium constant associated to the $\mathrm{At}^{+}+\mathrm{I}^{-}+\mathrm{Br}^{-} \rightleftharpoons \mathrm{IAtBr}^{-}$reaction is determined to be $10^{7.5 \pm 0.2}$, which is in fair agreement with the density functional theory predicted one $\left(10^{6.9}\right)$. This system not only constitutes the very first example of a ternary trihalogen species that involves the astatine element, but is also the first trihalogen species reported to predominate in solution. Also, we show that the oxidation number of At is zero in this species, as in the other molecules and anions that $\mathrm{At}^{+}$can form with $\mathrm{Cl}^{-}, \mathrm{Br}^{-}$ and $\mathrm{I}^{-}$ligands.
\end{abstract}

Evidencing and characterizing the structures and properties of new chemical compounds usually requires to perform a wide range of complementary experiments, among which spectroscopy ones play a major role. However, in some particular cases, one can only work with quantities of matter that prohibit the use of such golden experiments. This is typically the case with the astatine (At, $Z=85$ ) element, which is artificially produced with the help of cyclotron facilities ${ }^{[1],[2]}$. Things can even become more complicated if only a tiny experimental domain admits the occurrence of the speculated chemical species in sufficient quantity. In this work, we demonstrate that quantum mechanical calculations can a priori guide the experimental study by predicting the experimental domain to be targeted. For this, the expected amounts of the chemical species to be evidenced should be sufficient to result in sizeable changes on the scrutinized "macroscopic" property (here the distribution coefficient of At in biphasic systems). Following this original approach, we show that it becomes possible to identify exotic species that may not have been evidenced otherwise.

It is of fundamental interest for chemists to characterize the chemical species that involve the At element, since some of its compounds may not have any analogue. Indeed, due to relativistic effects, the behavior of At may be very different to its nearest halogen, iodine. For instance, the Pourbaix diagram

[a] N. Guo, D.-C. Sergentu, Dr. D. Teze, Dr. J. Champion, Dr. G. Montavon, Dr. R. Maurice SUBATECH, UMR CNRS 6457

IN2P3/Mines Nantes/Université de Nantes

4 rue Alfred Kastler, BP 20722, 44307 Nantes Cedex 3, France

E-mail: remi.maurice@subatech.in2p3.fr

[b] D.-C. Sergentu, Dr. N. Galland

CEISAM, UMR CNRS 6230

Université de Nantes

2 rue de la Houssinière, BP 92208, 44302 Nantes Cedex 3, France

E-mail: nicolas.galland@univ-nantes.fr

Supporting information for this article is given via a link at the end of the document.
$(E-\mathrm{pH})$ of astatine involves cationic species such as $\mathrm{At}^{+}$and $\mathrm{AtO}^{+[3],[4],[5]}$, while the one of iodine only involves anionic or neutral species. Of course, in some cases, At and I behave in a similar way. Since the ternary trihalogen $\mathrm{BrICl}^{-}$species has been characterized in solution by means of voltametric studies ${ }^{[6]}$, and its photodissociation behavior in the gas phase studied by means of pump-probe experiments ${ }^{[7]}$, we hypothesized that the $\mathrm{IAtBr}^{-}$analogue would form in solution. This work aimed at evidencing the realness of this formation, notably though the determination of the equilibrium constant $(K)$ associated to the following reaction:

$\mathrm{At}^{+}+\mathrm{I}^{-}+\mathrm{Br}^{-} \rightleftharpoons \mathrm{IAtBr}^{-}$

However, the presence of the $\mathrm{I}^{-}$and $\mathrm{Br}^{-}$ions can lead to the formation of binary dihalogen molecules and binary trihalogen anions that would compete with the formation of the targeted ternary trihalogen species. These formations are associated with the following reactions:

$\mathrm{At}^{+}+\mathrm{X}^{-} \rightleftharpoons \mathrm{AtX}$

$\mathrm{At}^{+}+2 \mathrm{X}^{-} \rightleftharpoons \mathrm{AtX}_{2}^{-}$

where $\mathrm{X}$ is either $\mathrm{Br}$ or $\mathrm{I}$, and $\beta_{1, \mathrm{X}^{-}}$and $\beta_{2, \mathrm{X}^{-}}$are the corresponding equilibrium constants associated with the involvement of one or two $X^{-}$units, respectively. Note that $K$ notations are used for the formations of ternary complexes and $\beta$ ones for the formations of binary $1: 1$ and $1: 2$ ones. Experimental values for the $\beta_{1, x^{-}}$and $\beta_{2, x^{-}}$constants are available in the literature, based on competition $(\mathrm{X}=\mathrm{Br})^{[8]}$ or electromigration ( $\mathrm{X}$ $=1)^{[9]}$ methods.

Table 1. Thermodynamic constants at zero ionic strength for the reactions of the $\mathrm{At}^{+}$cation with $\mathrm{Br}^{-}$and $\mathrm{I}^{-}$ligands (from the literature).

\begin{tabular}{lll}
\hline $\mathrm{X}^{-}$ & $\mathrm{Br}^{-}$ & $\mathrm{I}^{[\text {[a] }}$ \\
\hline $\log \beta_{1, \mathrm{X}^{-}}$ & $3.0 \pm 0.2^{[8]}$ & $6.0^{[9]}$ \\
$\log \beta_{2, \mathrm{X}^{-}}$ & $4.1 \pm 0.3^{[8]}$ & $9.2^{[9]}$ \\
\hline
\end{tabular}

[a] Extrapolated from the values at $0.5 \mathrm{M}$ ionic strength (6.2 and 9.4, respectively) to zero ionic strength through a truncated Davies equation ${ }^{[10]}$.

Quantum mechanical calculations can be used as a costeffective tool for assessing the stability of $\mathrm{IAtBr}^{-}$with respect to the other dihalogen and trihalogen species of interest. To accurately predict equilibrium constants by means of relativistic density functional theory (DFT), one can make use of ligandexchange reactions in order to take profit of error cancellations $^{[3,5,8,11]}$. Since experimental $\log \beta_{2, X^{-}}$values are available for $X=B r$ and $X=1$, we consider here the following reaction:

$\frac{1}{2} \mathrm{AtBr}_{2}{ }^{-}+\frac{1}{2} \mathrm{Atl}_{2}{ }^{-} \rightleftharpoons \mathrm{IAtBr}^{-}$

The computation of the associated equilibrium constant, $K_{1}$, readily leads to the Log $K$ value of interest as follows:

$\log K=\frac{1}{2} \log \beta_{2, \mathrm{Br}^{-}}+\frac{1}{2} \log \beta_{2, \mathrm{I}^{-}}+\log K_{1}$ 
The B3LYP exchange-correlation functional ${ }^{[12]}$ was used, solvation free energies were estimated by means of a conductor-like polarizable continuum model $(\mathrm{CPCM})^{[13]}$, and other computational details that typically lead to accurate complexation constants in the field of astatine chemistry were chosen ${ }^{[3,5,8,11]}$ (see Supporting Information for more details and ref 11 for an extensive benchmark). A Log $K_{1}$ value of 0.2 was computed, leading to a predicted Log $K$ value of 6.9. Using the latter combined with the equilibrium constants reported in Table 1 , we can elaborate a predictive speciation diagram of At in aqueous phase with the presence of both the $\mathrm{I}^{-}$and $\mathrm{Br}^{-}$anions (see Figure 1). It becomes clear from this prediction that a predominance domain does exist for the $\mathrm{IAtBr}^{-}$species, but that it should be rather small. Indeed, according to the theoretical prediction, $\mathrm{IAtBr}^{-}$could be a predominant species when the concentrations of $\mathrm{I}^{-}$is within the $\sim 10^{-4}$ to $\sim 10^{-2} \mathrm{M}$ range meanwhile the $\mathrm{Br}^{-}$one is within the $\sim 0.1$ to $\sim 1 \mathrm{M}$ one (see Figure 1). This severely restricts the experimental domain to be covered. Additional experimental constraints may further be considered.

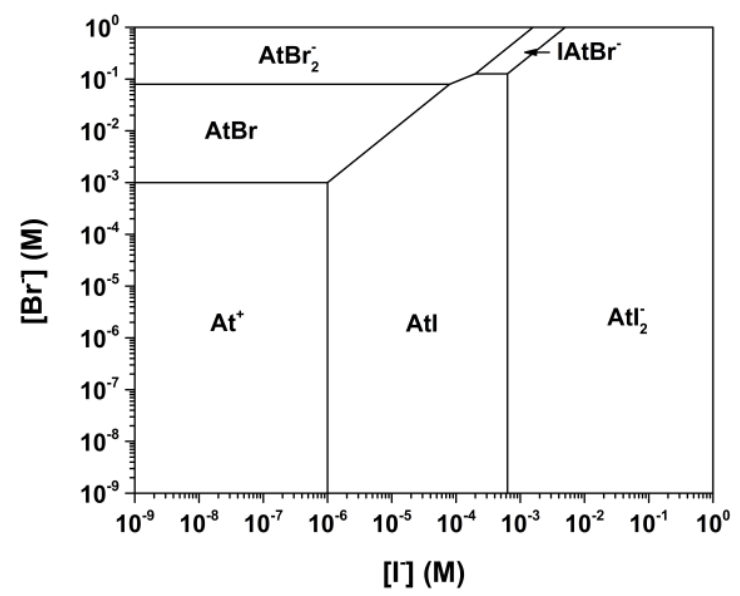

Figure 1. Predicted speciation diagram of At as a function of the initial concentrations of both the $\mathrm{Br}^{-}$and $\mathrm{I}^{-}$anions, based on the parameters displayed in Table 1 and the DFT predicted Log $K$ value.

A competition method was used to experimentally investigate the speciation of At in the aqueous phase. In this work, we consider the distribution coefficient $(D)$ between two liquid phases, one aqueous and the other organic (the solvent being the toluene $)^{[14]}$. Although a change in the $D$ value arising from a change in experimental conditions must indicate a change in speciation, an apparent constant $D$ value may hide some composition changes in each phase. Therefore, one must find conditions that lead to sizeable changes in $D$ to evidence new species. Simulations of the distribution coefficient as a function of the initial $\mathrm{I}^{-}$concentration at fixed $\mathrm{Br}^{-}$concentrations were conducted (see Figure 2). Four $\mathrm{Br}^{-}$concentrations were considered, the largest value corresponding to the limit of validity of the truncated Davies equation, and the smallest one being out of the predicted predominance domain of $\mathrm{IAtBr}^{-}$. We then select experimental conditions for which significant deviations appear between the model curves obtained by only considering the binary species (dash lines) and the ones obtained by also considering the ternary anion (solid lines). Therefore, we have retained the 0.1 and $0.05 \mathrm{M}$ initial concentrations for the $\mathrm{Br}^{-}$ligand, and we vary the $\mathrm{I}^{-}$one between $5 \times 10^{-5}$ and $2.5 \times 10^{-4} \mathrm{M}$ (i.e. we start near the beginning of the expected deviation and we end close to the maximum of it).

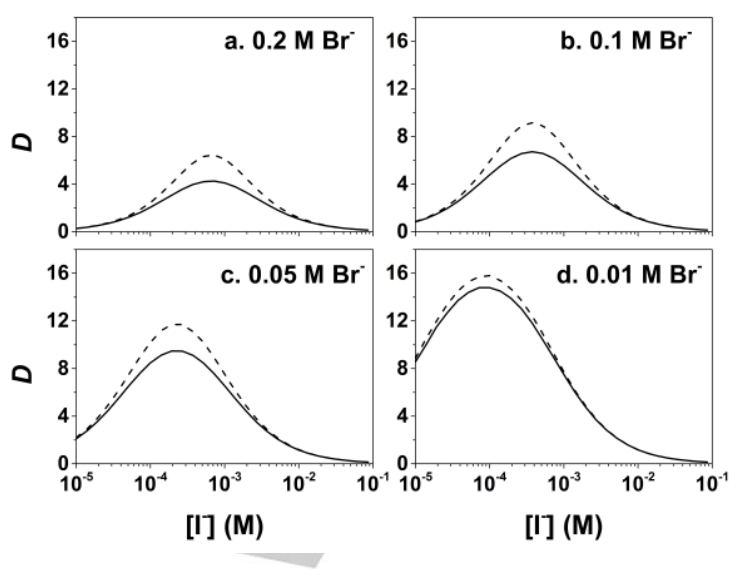

Figure 2. Simulation curves of the biphasic system: distribution coefficient $(D)$ of astatine as a function of the $\mathrm{I}^{-}$initial concentration at fixed $\mathrm{Br}^{-}$ concentrations (a.: $0.2 \mathrm{M}$, b.: $0.1 \mathrm{M}$, c.: $0.05 \mathrm{M}$, d.: $0.01 \mathrm{M}$ ), based on the parameters displayed in Table 1 and the DFT predicted Log $K$ value. Dash lines correspond to the simulations that only deal with the binary $1: 1$ and $1: 2$ species, while solid lines are used for the simulations that also account for the formation of the $\mathrm{IAtBr}^{-}$species in the aqueous phase.

Prior to study ternary media that a priori include $\mathrm{At}^{+}, \mathrm{I}^{-}$and $\mathrm{Br}^{-}$ ions, we start by revisiting the binary $\mathrm{At}^{+} / \mathrm{Br}^{-}$and $\mathrm{At}^{+} / \mathrm{I}^{-}$systems to get accurate and consistent (with the same experimental procedure) values of the $\beta_{1, x^{-}}$and $\beta_{2, x^{-}}$constants. The aqueous phase consists in $0.1 \mathrm{M}$ of $\mathrm{HClO}_{4}(\mathrm{pH} \approx 1, E \approx 0.6 \mathrm{~V})$, in which $\mathrm{At}^{+}$is the astatine dominant species, in the absence of complexing agent, according to its Poubaix diagram ${ }^{[3],[4],[5]}$. The modelling of the $\mathrm{At}^{+} / \mathrm{Br}^{-}$system with adjustable $\log \beta_{1, \mathrm{Br}^{-}}$and $\log \beta_{2, \mathrm{Br}^{-}}$ parameters (see Supporting Information) leads to the values displayed in Table 2. These values are in a good agreement with the previously published data ${ }^{[8]}$. Table 2 also gives the thermodynamic constants associated with the $\mathrm{At}^{+} / \mathrm{I}^{-}$binary system, which were derived from the modelling of the At distribution coefficient as a function of $\mathrm{I}^{-}$concentration (see Figure 3). The experimental data shows no signature of any speciation change when the initial $\mathrm{I}^{-}$concentration is below $10^{-8}$ $\mathrm{M}$. The increase of $D$ value between $10^{-8}$ and $10^{-5} \mathrm{M}$ implies the formation of a new species that is extracted in toluene, and a plateau is reached between $10^{-5}$ and $10^{-3} \mathrm{M}$. The $D$ value then decreases, which suggests the formation of another species that is not extracted in toluene. The experimental data are successfully modelled by considering the formation of 1:1 and 1:2 complexes, and the determined thermodynamic constants are found to be in good agreement with the electromigration data. $^{[9]}$ 
Table 2. Thermodynamic constants at zero ionic strength for the reactions of the $\mathrm{At}^{+}$cation with $\mathrm{Br}^{-}$and $\mathrm{I}^{-}$ligands (this work).

\begin{tabular}{lll}
\hline $\mathrm{X}^{-}$ & $\mathrm{Br}^{-}$ & $\mathrm{I}^{-}$ \\
\hline $\log \beta_{1, \mathrm{X}^{-}}$ & $2.7 \pm 0.2$ & $6.1 \pm 0.2$ \\
$\log \beta_{2, \mathrm{X}^{-}}$ & $3.8 \pm 0.3$ & $8.8 \pm 0.2$ \\
\hline
\end{tabular}

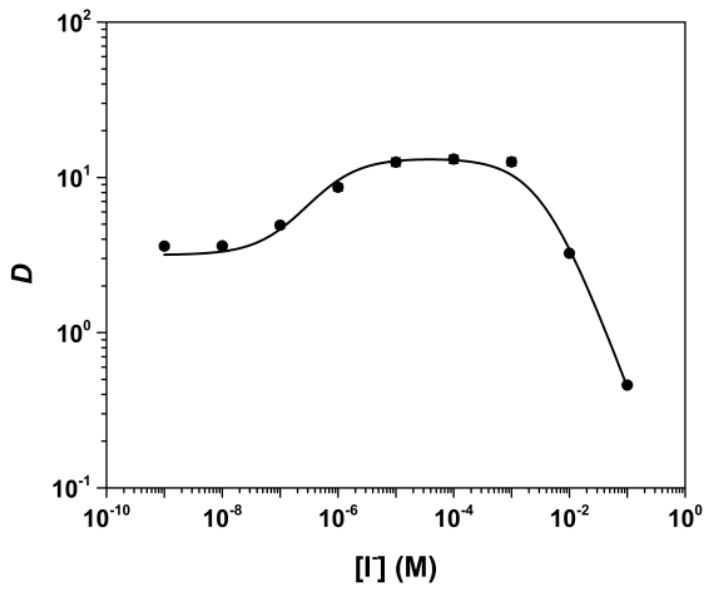

Figure 3. $\mathrm{At}^{+} / \mathrm{I}^{-}$binary system: distribution coefficient as a function of the initial $\mathrm{I}^{-}$concentration. The model curve obtained with the $\log \beta_{1, \mathrm{I}^{-}}$and $\log \beta_{2, \mathrm{I}^{-}}$ values reported in Table 2 is displayed in solid line.

We now continue by analysing the data obtained for the ternary $\mathrm{At}^{+} / \mathrm{I}^{-} / \mathrm{Br}^{-}$system. As shown in Figure 4 , the $D$ value increases

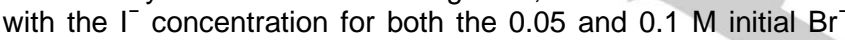
concentrations. First, we tried to model the data by only considering binary $1: 1$ and $1: 2$ species with the parameter values that are given in Table 2. As can be seen, the model

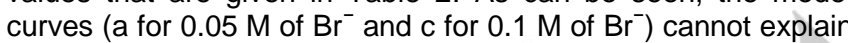
at all the experimental data. Therefore, the formation of at least another species must be considered. Following the previous prediction, we then further consider the formation of the $\mathrm{IAtBr}^{-}$ species via reaction 1. As can be seen in Figure 4 (curve $b$ for

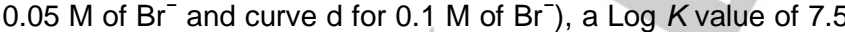
leads to a satisfactory modelling of the experimental data. Furthermore, the fairly good agreement between the experimental value of $\log K(7.5 \pm 0.2)$ and the theoretical one (6.9) prompt us to confirm the existence of the $\mathrm{AtBr}^{-}$species. The introduction of the $\mathrm{IAtBr}^{-}$species in the model leads to an effective decrease in the estimated Atl population (see Figure S2). Since this species can be extracted in toluene, contrary to the anionic $1 \mathrm{AtBr}^{-}$one, the formation of the $\mathrm{AtBr}^{-}$species translates in Figure 4 in a decrease in the modelled distribution coefficient.

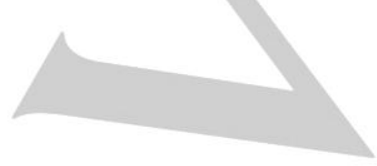

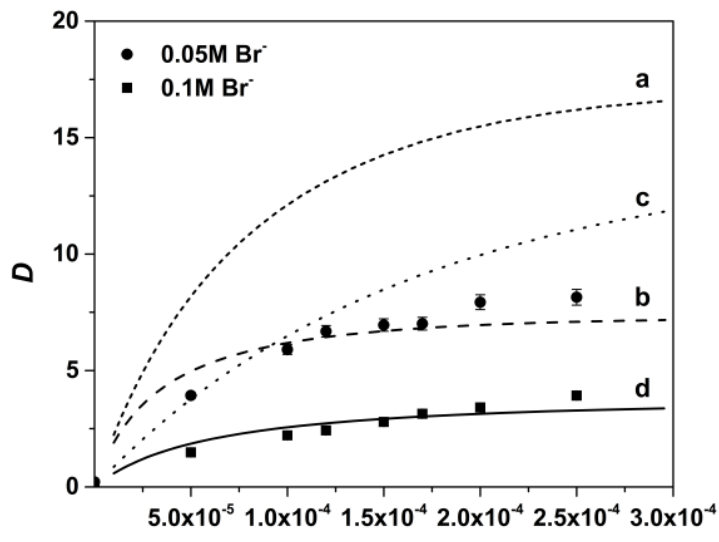

[I] (M)

Figure 4. $\mathrm{At}^{+} / \mathrm{I}^{-} / \mathrm{Br}^{-}$ternary system: distribution coefficient as a function of the

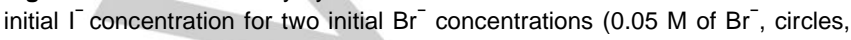

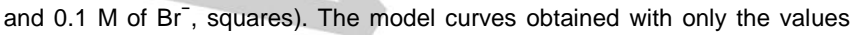

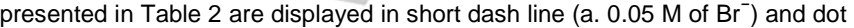

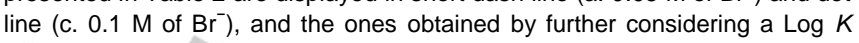

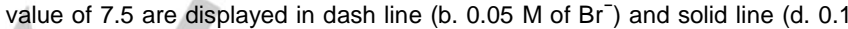
$\mathrm{M}$ of $\mathrm{Br}^{-}$).

Having experimentally determined the Log $K$ value, we can now build the At speciation diagram as a function of both the initial concentrations of $\mathrm{I}^{-}$and $\mathrm{Br}^{-}$(see Figure 5). The $\mathrm{IAtBr}^{-}$species can actually predominate for concentrations of $\mathrm{I}^{-}$around $10^{-5}-10^{-2} \mathrm{M}$ and $\mathrm{Br}^{-}$around $0.1-1 \mathrm{M}$. This species, being the heaviest possible ternary trihalogen one, deserves some more attention from a chemical point of view. Of course, no structural information can be experimentally obtained on this species. Therefore, we discuss its relativistic DFT structure. All the structures of the trihalogen species considered in this work, i.e. $\mathrm{AtBr}_{2}{ }^{-}, \mathrm{Atl}_{2}{ }^{-}$, and $\mathrm{IAtBr}^{-}$, are linear, the At atom being located in between the other two halogen ones. Therefore, the $\mathrm{IAtBr}^{-}$ species is analogous to the ternary trihalogen $\mathrm{BrICl}^{-}$species, for which the heavier halogen, $I$, is located in between the two lighter ones ${ }^{[7]}$. The At-X distances are $2.91 \AA\left(\mathrm{AtBr}_{2}{ }^{-}\right), 3.15 \AA$ $\left(\mathrm{Atl}_{2}{ }^{-}\right)$, and 2.92 and $3.14 \AA\left(\mathrm{ItBr}^{-}\right)$at the considered relativistic DFT level. One should stress that spin-orbit coupling must be taken into account for obtaining accurate geometries, At-X distances being affected by up to $\sim 0.1 \AA$ in these systems. To determine the oxidation numbers (ONs) of At in these species, and also in the AtBr and Atl ones, as well as the ON of I in $\mathrm{BrICl}^{-}$, we have estimated the number of valence $p$ electrons of the heaviest halogen with complete active space configuration interaction $(\mathrm{CASCl})$ calculations for which previously obtained complete active space self-consistent field (CASSCF) active orbitals were localized (see Supporting Information for more details). In all these species, more than 4.8 valence $p$ electrons are obtained for the heaviest halogen, meaning that the actual wave function is much closer to the neutral limit for this halogen (5 $p$ electrons) than to the first ionized one ( $4 p$ electrons). In other words, the ON of At in $\mathrm{AtBr}, \mathrm{Atl}, \mathrm{AtBr}_{2}{ }^{-}, \mathrm{Atl}_{2}{ }^{-}$and $\mathrm{IAtBr}{ }^{-}$is zero (note that the $\mathrm{ON}$ of $\mathrm{I}$ in $\mathrm{BrICl}^{-}$is actually also zero), contrary to what was previously assumed for the Atl and $\mathrm{Atl}_{2}{ }^{-}$ 
cases $^{[9]}$. Since these species were formed from the $\mathrm{At}^{+}$moiety, one can consider that the formations of the molecules and ions considered in this work lead to a change in the At ON. This reveals that the wave functions in these species are not dominated by ionic configurations (of the forms $\mathrm{At}^{+} \mathrm{X}^{-}$and $\mathrm{X}^{-}$ $\mathrm{At}^{+} \mathrm{Y}^{-}$, respectively), but rather by covalent ones (of the forms At $-X$ and $X-A t Y^{-}$and $X^{-} A t-Y$, respectively). Note that, as expected, test calculations showed that the inclusion of spinorbit coupling hardly affects the number of At $p$ electrons, and thus, cannot change our conclusions on the At ON.

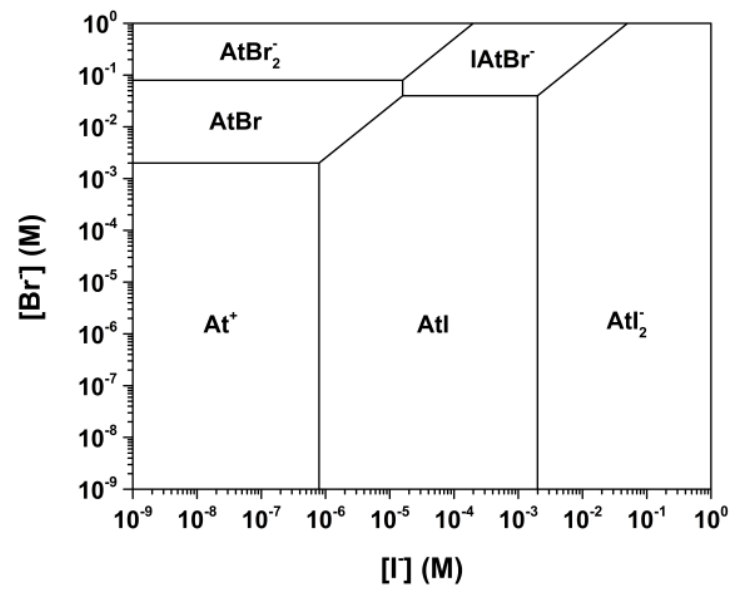

Figure 5. Experimental speciation diagram of At as a function of the initial concentrations of both the $\mathrm{Br}^{-}$and $\mathrm{I}^{-}$anions, based on the parameters displayed in Table 2 and the experimentally determined Log $K$ value of 7.5 .

In the present work, we have shown that adequate computational predictions can lead to the experimental discovery of an exotic chemical species, namely $\mathrm{IAtBr}^{-}$. Of course, it is of high interest for chemists to know if other ternary trihalogen species that involve the At element can exist or even predominate in solution. Using the in silico methodology introduced in this work, we have assessed the possibility of forming the $\mathrm{AtCl}^{-}$anion in the ternary $\mathrm{At}^{+} / \mathrm{I}^{-} / \mathrm{Cl}^{-}$case (see Supporting Information). Based on this prediction, we conclude that $\mathrm{IAtCl}^{-}$can be formed in solution. However, considering the extremely small-predicted domain of predominance, the experimental evidence of its existence may not be reachable in practice. Therefore, it is not yet confirmed if any other ternary trihalogen species involving At can predominate in solution.

\section{Acknowledgements}

This work was supported by grants funded by the French National Agency for Research with "Investissements d'Avenir" (ANR-11EQPX-0004, ANR-11-LABX-o018). It was carried out using HPC resources from GENCI-CINES/IDRIS (grant 2015-C2015085117) and CCIPL ("Centre de Calcul Intensif des Pays de la Loire").

Keywords: ternary trihalogen species $\cdot$ competition experiments - density functional theory $\cdot$ theoretical prediction $\cdot$ astatine

[1] M. R. Zalutsky, M. Pruszynski, Curr. Radiopharm. 2011, 4, 177-85.

[2] D. S. Wilbur, Nat. Chem. 2013, 5, 246.

[3] D.-C. Sergentu, D. Teze, A. Sabatié-Gogova, C. Alliot, N. Guo, F. Bassal, I. Da Silva, D. Deniaud, R. Maurice, J. Champion, et al., Chem. A Eur. J. 2016, 22, 2964-2971.

[4] J. Champion, C. Alliot, E. Renault, B. M. Mokili, M. Chérel, N. Galland, G. Montavon, J. Phys. Chem. A 2010, 114, 576-82.

[5] J. Champion, A. Sabatié-Gogova, F. Bassal, T. Ayed, C. Alliot, N. Galland, G. Montavon, J. Phys. Chem. A 2013, 117, 1983-90.

[6] R. Guidelli, F. Pergola, J. Inorg. Nucl. Chem. 1969, 31, 1373-1381.

[7] A. Sanov, T. Sanford, L. J. Butler, J. Vala, R. Kosloff, W. C. Lineberger, J.Phys.Chem. 1999, 103, 10244-10254.

[8] J. Champion, M. Seydou, A. Sabatié-Gogova, E. Renault, G. Montavon, N. Galland, Phys. Chem. Chem. Phys. 2011, 13, 1498492.

[9] R. Ludwig, S. Fischer, H. Hussein, M. Frind, R. Dreyer, J. Radioanal. Nucl. Chem. Artic. 1989, 134, 141-149.

[10] B. J. Colston, V. J. Robinson, J. Environ. Radioact. 1995, 29, 121136.

[11] D.-C. Sergentu, G. David, G. Montavon, R. Maurice, N. Galland, J. Comput. Chem. 2016, 37, 1345-54.

[12] P. J. Stephens, F. J. Devlin, C. F. Chabalowski, M. J. Frisch, J. Phys. Chem. 1994, 98, 11623-11627.

[13] M. Cossi, N. Rega, G. Scalmani, V. Barone, J. Comput. Chem. 2003 , 24, 669-681.

[14] J. Champion, C. Alliot, S. Huclier, D. Deniaud, Z. Asfari, G. Montavon, Inorganica Chim. Acta 2009, 362, 2654-2661. 


\section{Entry for the Table of Contents}

\section{COMMUNICATION}

Relativistic density functional theory is used by Guo et al. in their Communication on page $x x x \mathrm{ft}$. to predict the tiny experimental domain in which it may be possible to detect the heaviest possible trihalogen species, $\mathrm{IAtBr}^{-}$. Competition experiments are then performed at ultra-trace concentrations, for (i) confirming the theoretical prediction and (ii) determining experimentally the associated reaction equilibrium constant.

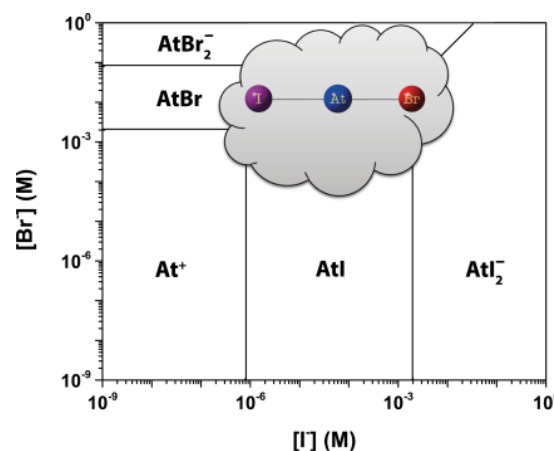

Ning Guo, Dumitru-Claudiu Sergentu, Dr. David Teze, Dr. Julie Champion, Dr. Gilles Montavon, Dr. Nicolas Galland* and Dr. Rémi Maurice*

\section{Page No. xxx - Page No. xxx}

The heaviest possible ternary trihalogen species, $\mathrm{IAtBr}^{-}$, evidenced in aqueous solution: An experimental performance driven by computations 